\title{
Aspectos socio demográficos y calidad de vida en salud Bucal, del personal del municipio de Azogues - Ecuador
}

\author{
Socio-demographic aspects and quality of life in oral health of the staff of the municipality of Azogues - Ecuador
}

\begin{abstract}
Cristina Dominguez-Crespo ${ }^{1, a, b, c,}$, Cristina Crespo-Crespo ${ }^{1, a, c, d}$, FernandoVallejo-Aguirre ${ }^{1, a, c, e,}$, Carmen Garcia-Rupaya ${ }^{2, a, c, e,}$, Alexis Evangelista-Alva ${ }^{3, a, c}$, Cesar del Castillo- López ${ }^{\text {4,a,f,g }}$
\end{abstract}

\section{RESUMEN}

Objetivo: Asociar los aspectos socio demográficos y la calidad de vida en salud bucal del personal del Municipio de Azogues - Ecuador. Material y métodos: La presente constituye una investigación transversal, descriptiva, observacional misma que investigó a 185 adultos, aplicándoles una encuesta para determinar aspectos relacionadas a datos personales, determinantes sociales y uso de servicio odontológico. Seguido se indagó sobre el impacto de la salud bucal en la calidad de vida utilizando el cuestionario validado Ohip -14Sp. Se efectúo análisis univariado y bivariado. Para el estudio bivariado se utilizó el Odds Ratio (OR) para asociar los factores mencionados con la calidad de vida relacionada a la salud bucal (CVRSB). Se contó con un nivel de confianza del 95\% y un $p<0,05$. Resultados: No se encontró asociación estadísticamente significativa al evaluar todas las variables con CVRSB; el grupo con la mayor prevalencia de impactos según el nivel de instrucción fueron Universitarios con un 46\%; según el ingreso mensual, los de ingreso mensual menor a $\$ 1000$ con un $66 \%$; de acuerdo a la denominación laboral los trabajadores con un 53\%; de acuerdo al motivo de consulta, fueron otras causas 39\%. Sin embargo, a pesar de que no hay una asociación significativa, se puede apreciar que el sexo masculino fue el más impactado. Conclusiones: Las determinantes sociales y CVRSB no estuvieron asociados. Las dimensiones limitación funcional, dolor físico y obstáculos fueron las más frecuentes.

PALABRAS CLAVE: calidad de vida, salud bucal, adultos, Condiciones Sociales.

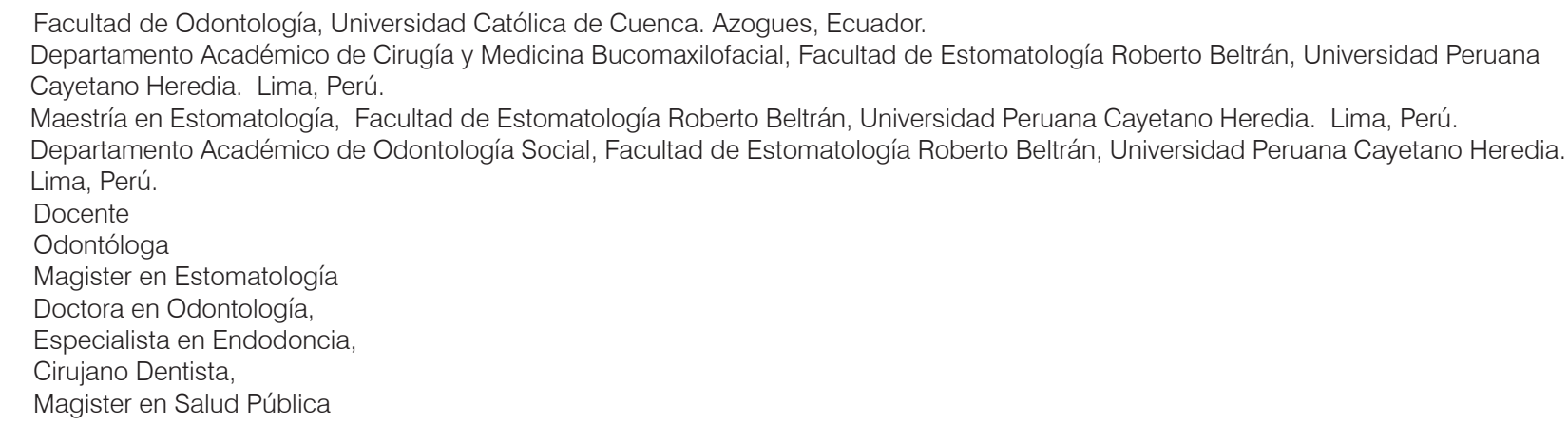




\section{SUMMARY}

Objective: To associate the socio-demographic aspects and the quality of life in oral health of the personnel of the Municipality of Azogues - Ecuador. Material and methods: This is a transversal, descriptive, observational research that investigated 185 adults, applying a survey to determine aspects related to personal data, social determinants and use of dental service. Afterwards, the impact of oral health on the quality of life was investigated using the Ohip-14Sp validated questionnaire. Univariate and bivariate analyzes were carried out. For the bivariate study, the Odds Ratio (OR) was used to associate the aforementioned factors with the quality of life related to oral health (HRQOL). There was a confidence level of $95 \%$ and $p<0.05$. Results: No statistically significant association was found when evaluating all variables with CVRSB; The group with the highest prevalence of impacts according to the level of education were University students with 46\%; according to the monthly income, the monthly income less than $\$ 1000$ with $66 \%$, according to the labor denomination the workers with 53\%; according to the reason for consultation, other causes were $39 \%$. However, although there is no significant association, it can be seen that the male sex was the most impacted. Conclusions: The social determinants and CVRSB were not associated. The dimensions of functional limitation, physical pain and obstacles were the most frequent.

\section{KEYWORDS: Quality of life, oral health, adults, social conditions.}

\section{INTRODUCCIÓN}

En Ecuador las políticas públicas están enmarcadas en el régimen de desarrollo y del buen vivir, dicha situación ha creado, un impacto significativo dentro de los diferentes grupos sociales gracias a reformas y políticas ejecutadas. Por otro lado, es conocido que el Sistema de Salud de Ecuador se caracteriza por el fraccionamiento en sectores, público, privado y mixto (1). Por su parte un sistema de seguro social costeado por importes de quienes vienen laborando en el sector formal cohabita con estructuras del subsistema privado que atiende a un grupo con mayor poder adquisitivo y con otro subsistema que atiende a los más pobre. Estudios refieren que existen barreras de carácter económico, geográfico y cultural que imposibilitan la llamada cobertura universal de salud, volviendo caótico el acceso al sistema. Asimismo, es importante indicar que ciertas dolencias orales constituyen un motivo de preocupación para los organismos universales reguladores de la salud pública; por ello, es preocupante que exista desinformación y poca importancia en relación a la salud bucal en el Ecuador; así como tampoco políticas públicas que garanticen coberturas adecuadas a los diferentes grupos poblacionales (1).

En Ecuador las cifras de prevalencia de caries dental son determinantes para evidenciar la gravedad del problema; pues según el informe reportado en el manual de regulación del Sistema Nacional de Salud del Área de Odontología de Ecuador 2010 menciona que el 88,2\% de los escolares tienen caries dental, y el CPOD (promedio de piezas definitivas con caries, perdidas u obturadas) es de 0,22 a los 6 años de esta manera la prevalencia tiene un incremento rápido a medida que avanza la edad, así , a los 8 años se cuenta con un promedio de 0,77 ; a los 12 años de 2,95 y a los 15 años el CPOD aumenta súbitamente a 4,65. Dicho estudio fue llevado a cabo por la ex dirección nacional de estomatología (2).

Por otra parte, a partir del año 1995 se introdujeron reformas en el sector salud, cuyo objetivo fue generar separación y traspaso de funciones del Ministerio de Salud Pública a las Municipalidades que lo requieran; esta implementación trajo consigo ciertos desacuerdos que lamentablemente repercutieron en: la calidad de atención, coberturas de servicios, especialmente afectándose sectores vulnerables (3).

Siendo entonces la calidad de vida una variable multidimensional; lleva consigo diferentes aspectos tales como situaciones biológicas, sociales, culturales y psicológicas, y si alguna de ellas se afectare traería como consecuencia una afectación de sus desempeños diarios. En este contexto se puede afirmar que una dolencia física de cualquier índole contribuiría a desmejorar las condiciones de vida del individuo que serán manifestadas de acuerdo a su autopercepción. En el transcurso del tiempo algunas investigaciones han demostrado la relevancia de los aspectos socio demográficos entre ellos salario, nivel de 
educación, situación laboral, que se relacionan con los indicadores de salud. De otro lado situaciones emocionales afectan negativamente la sensación de sentirse bien, al punto que se considera al estrés como una de las causas de un déficit en la calidad de vida de las personas (4).

El objetivo del presente estudio fue determinar asociación entre los aspectos socios demográficos y calidad de vida en salud oral del personal del Municipio de Azogues - Ecuador 2015.

\section{MATERIAL Y MÉTODOS}

El diseño fue de corte transversal observacional descriptivo. La población constó de 700 individuos con edades de 20 a 64 años, con dependencia laboral municipal. La muestra quedó constituida por 185 trabajadores y empleados, los criterios de selección fueron tener entre 20 a 64 años, que estén en capacidad de entender el cuestionario y firmar el consentimiento informado. El muestreo fue probabilístico aleatorio simple (5).

Se procedió con una socialización del objetivo y naturaleza del estudio al personal implicado y se aseguró la participación de los mismos mediante la firma del consentimiento informado. Luego se recolectaron los datos de lunes a viernes en horario de labores.

Se usó el instrumento validado OHIP-14Sp en español, que incluía 7 dimensiones: limitación funcional, dolor físico, aflicción sicológica, incapacidad física, incapacidad sicológica, incapacidad social y obstáculos (6).

El procedimiento se resume de la siguiente manera: en primer lugar, se registraron los datos generales del sujeto de investigación, en segundo lugar, se determinaron aspectos sociodemográficos como nivel educativo, designación laboral, ingreso económico, tipo de servicio dental, tiempo desde la última visita al dentista, motivo de consulta odontológica; y finalmente se aplicó el instrumento que estimó el perfil de impacto en salud oral. Por otro lado, se establecieron coordinaciones con el director departamental de Recursos Humanos quien facilitó el proceso, de modo que no interrumpa las actividades diarias preestablecidas de los trabajadores. El tiempo estimado de duración por encuesta fue de 6 a 8 minutos aproximadamente las mismas se desarrollaron en diferentes departamentos de la municipalidad de acuerdo al cronograma preestablecido, asegurando manejar un número aproximado de 15 adultos en el día.

Con el fin de evaluar la severidad del impacto cada pregunta del instrumento posee un peso derivado de la técnica de comparación pareada de Thusrtone, la escala de valoración se empleó fue 0 nunca; 1 casi nunca; 2 ocasionalmente; 3 frecuentemente y 4 muy frecuentemente $(7,8)$.

Para determinar el impacto se efectuó la suma del puntaje obtenido en las 14 preguntas donde el valor de 0 (no impacto) a 14 (máximo impacto). Las derivaciones se alcanzaron a través de la separación de los participantes de acuerdo a los cuartiles de la distribución de la muestra. Los que están en el último cuartil (75\%) fueron considerados como de mayor impacto. Para el análisis se utilizó el programa SPSS versión 22 , la estadística descriptiva se presentó mediante la obtención de las frecuencias absolutas y relativas, y la obtención de los promedios y desviación estándar. La estadística inferencial utilizó la Prueba de U de Mann Whitney para la comparación de variables numéricas y para determinar la asociación de variables se usó Odd Ratio (O.R.). El estudio contó con un nivel de confianza de $95 \%$ y un límite de significancia de $p<0,05$.

El estudio contó con la aprobación del Comité Institucional de Ética (CIE) de la Universidad Peruana Cayetano Heredia. Constancia 220-13-15 Aprobación 64159; debido a que el presente estudio corresponde a una tesis de maestría de la misma universidad.

\section{RESULTADOS}

Se evaluaron 185 personas, de las cuales el sexo masculino resultó con mayor frecuencia $(62,70 \%)$ se trata en su mayoría de una población joven de 20 a 40 años $(62,16 \%)$, Se distribuye de manera simétrica la designación laboral, siendo ligeramente mayor el grupo de trabajadores $(52,97 \%)$, en cuanto al nivel de instrucción predomina la formación universitaria $(45,40 \%)$; tiene mayor frecuencia el ingreso económico mensual menor a $\$ 1000(62,70 \%)$. El motivo de consulta se presentó de manera simétrica entre las tres categorías planteadas. En cuanto al uso de servicios odontoló- 
gicos, existe simetría en el tipo de servicio visitado, con una ligera preferencia por los servicios privados $(53,51 \%)$, finalmente respecto a la frecuencia para asistir al dentista es más frecuente el grupo de aquellos que visitan al odontólogo en períodos menores a un año $(57,83 \%)$ (tabla 1$)$.

La pregunta acerca de si ha estado preocupado por problema con dientes boca o prótesis dental fue la que obtuvo mayor frecuencia de respuestas de autopercepción entre frecuente y muy frecuentemente $16,21 \%$ y $10,81 \%$ respectivamente (tabla 2 ).

En cuanto a los dominios indagados por el OHIP$14 \mathrm{Sp}$ se encontró diferencias estadísticamente significativas al comparar entre ambos sexos, en las dimensiones dolor físico, siendo esta percepción mayor en mujeres de 20 a 40 años. En la comparación de los dominios evaluados de acuerdo a las edades, las dimensiones limitación funcional, dolor físico y obstáculos, presentaron diferencia estadísticamente significativa evaluada mediante U de Mann Withney $\mathrm{p}<0,05$ (tabla 3).
Por otro lado, no se encontró asociación estadísticamente significativa entre nivel de instrucción e impacto de la calidad de vida, $X^{2} p=0,4469$. En cuanto al ingreso mensual tampoco se encontró asociación estadísticamente significativa $\mathrm{X}^{2} \mathrm{p}=0,314$ y respecto a la denominación laboral no se encontró una asociación estadísticamente significativa O.R.=1,03 IC $95 \%(0,58$ - 1,85) (tabla 4).

No se encontró asociación estadísticamente significativa entre el motivo de consulta y el impacto en la CVRSB, $X^{2} \mathrm{p}=0,8181$; por otro lado, tampoco se encontró asociación entre la frecuencia de asistencia al dentista y el impacto a la CVRSB y en relación al tipo de servicios visitado, no se encontró asociación entre el tipo de servicio que se visita y el impacto a la CVRSB; O.R.= 0,69 IC 95\% (0,38 - 1,24) (tabla 5).

Finalmente, la edad y el sexo no resultaron estar asociadas con la calidad de vida presentando un OR de 1,4 IC $95 \%(0,79-2,67)$ y OR de 0,9 IC $95 \%(0,5$ y $1,7)$; respectivamente. Sin embargo, a pesar de que no hay una asociación significativa, se puede apreciar que el sexo masculino fue el más impactado (tabla 6). 
Tabla 1. Perfil Sociodemográfico del Personal del Municipio de Azogues

\begin{tabular}{|c|c|}
\hline Características Sociodemográficas & n (\%) \\
\hline \multicolumn{2}{|l|}{ Sexo } \\
\hline Masculino & $116(62,70)$ \\
\hline Femenino & $69(37,63)$ \\
\hline \multicolumn{2}{|l|}{ Edad en años } \\
\hline 20 a 40 & $115(62,16)$ \\
\hline 41 a 64 & $70(37,83)$ \\
\hline \multicolumn{2}{|l|}{ Nivel de Instrucción } \\
\hline Primaria & $24(12,97)$ \\
\hline Secundaria & $77(41,62)$ \\
\hline Universitario & $84(45,40)$ \\
\hline \multicolumn{2}{|l|}{ Ingreso económico mensual } \\
\hline Básico & $24(12,97)$ \\
\hline Menor a 1000 & $116(62,70)$ \\
\hline Mayor a 1000 & $45(24,32)$ \\
\hline \multicolumn{2}{|l|}{ Designación Laboral } \\
\hline Empleado & $87(47,02)$ \\
\hline Trabajador & $98(52,97)$ \\
\hline \multicolumn{2}{|l|}{ Motivo de Consulta al Dentista } \\
\hline Dolor & $59(31,89)$ \\
\hline Otras causas & $65(35,13)$ \\
\hline Rutina & $61(32,97)$ \\
\hline \multicolumn{2}{|l|}{ Tipo de Servicio Visitado } \\
\hline Publico & $86(46,48)$ \\
\hline Privado & $99(53,51)$ \\
\hline \multicolumn{2}{|l|}{ Frecuencia para asistir al dentista } \\
\hline Menos de un año & $107(57,83)$ \\
\hline Anual & $62(33,51)$ \\
\hline Más de tres años & $16(8,64)$ \\
\hline TOTAL & $185(100)$ \\
\hline
\end{tabular}


Tabla 2 . Distribución individual de respuestas del Ohip-14 en personal del Municipio de Azogues 2015

\begin{tabular}{|c|c|c|c|c|c|}
\hline Preguntas OHIP-14 & $0(\%)$ & $1(\%)$ & $2(\%)$ & $3(\%)$ & $4(\%)$ \\
\hline $\begin{array}{l}\text { Tiene usted dificultad para pronunciar al- } \\
\text { gunas palabras debido a problemas con sus } \\
\text { dientes boca o prótesis dental }\end{array}$ & $145(78.37)$ & $14(7,56)$ & $17(9,18)$ & $4(2,16)$ & $5(2,70)$ \\
\hline $\begin{array}{l}\text { Ha sentido usted que su sensación de gusto } \\
\text { ha empeorado debido a problemas con sus } \\
\text { dientes ,boca o prótesis dentales }\end{array}$ & $132(71.35)$ & $12(6,48)$ & $31(16,75)$ & $6(3,24)$ & $4(2,16)$ \\
\hline $\begin{array}{l}\text { Ha sentido Ud. incomodidad al comer al- } \\
\text { gunos alimentos debido a problemas con su } \\
\text { boca ,prótesis }\end{array}$ & $77(41.62)$ & $26(14,05)$ & $50(27,02)$ & $19(10,27)$ & $13(7,02)$ \\
\hline $\begin{array}{l}\text { Ha estado preocupado debido a problemas } \\
\text { con sus dientes, boca o prótesis dentales }\end{array}$ & $78(42.16)$ & $16(8,64)$ & $41(22,16)$ & $30(16,21)$ & $20(10,81)$ \\
\hline $\begin{array}{l}\text { Se ha sentido nervioso debido a problemas } \\
\text { con sus dientes, boca o prótesis dentales }\end{array}$ & $117(63,24)$ & $15(8,10)$ & $26(14,05)$ & $13(7,02)$ & $14(7,56)$ \\
\hline $\begin{array}{l}\text { Ha sido su dieta poco satisfacción debido a } \\
\text { problemas con sus dientes, boca o prótesis } \\
\text { dentales }\end{array}$ & $145(78.37)$ & $10(5,40)$ & $16(8,64)$ & $9(4,86)$ & $5(2,70)$ \\
\hline $\begin{array}{l}\text { Ha tenido usted que interrumpir sus comidas } \\
\text { debido a problemas con sus dientes, boca o } \\
\text { prótesis dentales }\end{array}$ & $143(77,29)$ & $14(7,56)$ & $19(10,27)$ & $6(3,24)$ & $3(1,62)$ \\
\hline $\begin{array}{l}\text { Encuentra dificultades para relajarse debido } \\
\text { a problemas con sus dientes, boca o prótesis }\end{array}$ & $149(80,54)$ & $11(5,94)$ & $16(8,64)$ & $2(1,08)$ & $7(3,78)$ \\
\hline $\begin{array}{l}\text { Se ha sentido perturbado frente a otras perso- } \\
\text { nas debido a problemas con sus dientes , boca } \\
\text { o prótesis dentales }\end{array}$ & $119(64,32)$ & $14(7,56)$ & $26(14,05)$ & $9(4,86)$ & $17(9,18)$ \\
\hline $\begin{array}{l}\text { Se ha sentido Ud. irritable con otras personas } \\
\text { debido a problemas con sus dientes, boca o } \\
\text { prótesis }\end{array}$ & $140(75,67)$ & $11(5,94)$ & $20(10,81)$ & $5(2,70)$ & $9(4,86)$ \\
\hline $\begin{array}{l}\text { Tiene Ud. dificultad para hacer sus trabajos } \\
\text { habituales debido a problemas con sus dien- } \\
\text { tes, boca o prótesis }\end{array}$ & $162(87,56)$ & $11(5,94)$ & $9(4,86)$ & $1(0,54)$ & $2(1,08)$ \\
\hline $\begin{array}{l}\text { Ha sentido Ud. que su vida es en general me- } \\
\text { nos satisfactoria debido a problemas con sus } \\
\text { dientes, boca o prótesis }\end{array}$ & $149(80,54)$ & $18(9,72)$ & $14(7,56)$ & $1(0,54)$ & $3(1,62)$ \\
\hline $\begin{array}{l}\text { Se ha sentido totalmente incapaz de funcio- } \\
\text { nar debido a problemas con sus dientes, boca } \\
\text { o prótesis dental }\end{array}$ & $169(91,35)$ & $5(2,70)$ & $8(4,32)$ & 0 & $3(1,62)$ \\
\hline
\end{tabular}


Tabla 3. Puntuaciones medias de los siete dominios del OHIP entre los sujetos de estudio según edad y sexo

\begin{tabular}{|c|c|c|c|c|c|c|c|c|c|c|}
\hline \multirow[t]{3}{*}{ Dimensiones } & \multicolumn{6}{|c|}{ Edad 20 a 40 años } & \multicolumn{4}{|c|}{ Edad 41 a 64 años } \\
\hline & \multicolumn{3}{|c|}{ Masculino $(n=66)$} & \multicolumn{3}{|c|}{ Femenino $(n=49)$} & \multicolumn{2}{|c|}{ Masculino $(n=50)$} & \multicolumn{2}{|c|}{ Femenino $(n=20)$} \\
\hline & Media & & D.E. & Media & & D.E. & Media & D.E. & Media & D.E. \\
\hline Limitación Funcional* & 0,83 & $*$ & 1,40 & 0,59 & & 1,00 & $1,60 *$ & 2,07 & 1,20 & 1,77 \\
\hline Dolor Físico & 1,68 & $*$ & 1,77 & 2,51 & $* *$ & 1,99 & $2,42 *$ & 2,04 & 2,20 & 2,35 \\
\hline Molestias Psicológicas & 2,05 & & 2,26 & 2,24 & & 2,21 & 2,80 & 2,53 & 2,25 & 2,69 \\
\hline Incapacidad Física & 0,65 & & 1,46 & 1,00 & & 1,54 & 1,10 & 2,05 & 1,20 & 2,19 \\
\hline Incapacidad Psicológica & 1,20 & & 1,74 & 1,37 & & 1,75 & 1,38 & 2,18 & 1,15 & 1,63 \\
\hline Incapacidad Social & 0,55 & & 1,13 & 0,96 & & 1,62 & 0,84 & 1,56 & 0,85 & 1,60 \\
\hline Obstáculos & 0,18 & $*$ & 0,63 & 0,37 & & 0,93 & $0,76 *$ & 1,53 & 1,30 & 2,20 \\
\hline
\end{tabular}

Tabla 4. Asociación entre condiciones sociales e impacto en la CVRSB

\begin{tabular}{|c|c|c|c|c|c|}
\hline \multirow[b]{2}{*}{ Determinantes } & \multicolumn{2}{|c|}{ Sin iImpacto } & \multicolumn{2}{|c|}{ Con impacto } & \multirow[b]{2}{*}{ Significancia } \\
\hline & $\mathbf{n}$ & $\%$ & $\mathbf{n}$ & $\%$ & \\
\hline \multicolumn{6}{|l|}{ Nivel de Instrucción } \\
\hline Primaria & 8 & 9,8 & 16 & 15,5 & \multirow{3}{*}{0,4469} \\
\hline Secundaria & 37 & 45,1 & 40 & 38,8 & \\
\hline Universitaria o Posgrado & 37 & 45,1 & 47 & 45,6 & \\
\hline \multicolumn{6}{|l|}{ Ingreso Mensual } \\
\hline Básico & 14 & 17,1 & 10 & 9,7 & \multirow{3}{*}{0,314} \\
\hline De 361 a 1000 & 48 & 58,5 & 68 & 66 & \\
\hline Mayor a 1000 & 20 & 24,4 & 25 & 24,3 & \\
\hline \multicolumn{6}{|l|}{ Denominación Laboral } \\
\hline Empleado & 39 & 47,6 & 48 & 46,6 & O.R. IC $95 \%$ \\
\hline Trabajador & 43 & 52,4 & 55 & 53,4 & $1,03(0,58-1,85)$ \\
\hline
\end{tabular}


Tabla 5. Uso de Servicios Odontológicos en relación a salud oral en personal del municipio de Azogues

\begin{tabular}{|c|c|c|c|c|c|c|}
\hline \multirow[t]{2}{*}{ Servicios Odontológicos } & & \multicolumn{2}{|c|}{$\underset{\text { Impacto }}{\text { Sin }}$} & \multicolumn{2}{|c|}{ Con Impacto } & \multirow[b]{2}{*}{ SIGNIFICANCIA } \\
\hline & & $\mathbf{n}$ & $\%$ & $\mathbf{n}$ & $\%$ & \\
\hline \multirow{3}{*}{ Motivo de Consulta al dentista } & Dolor & 26 & 31,7 & 33 & 32 & \multirow{3}{*}{0,8181} \\
\hline & Otras Causas & 25 & 30,5 & 40 & 39 & \\
\hline & Rutina & 31 & 37,8 & 30 & 29 & \\
\hline \multirow{3}{*}{ Frecuencia de Asistencia al Dentista } & Menos de 1 año & 46 & 56,1 & 61 & 59 & \multirow{3}{*}{0,1471} \\
\hline & Anual & 32 & 39 & 30 & 29 & \\
\hline & Más de 2 Años & 4 & 4,9 & 12 & 12 & \\
\hline \multirow[t]{2}{*}{ Tipo de Servicio Visitado } & Público & 34 & 41,5 & 52 & 51 & O.R. IC 95\% \\
\hline & Privado & 48 & 58,5 & 51 & 50 & $0,69(0.38-1.24)$ \\
\hline
\end{tabular}

Tabla 6. Asociación entre las variables demográficas y el impacto en la CVRSB.

\begin{tabular}{llcccccc}
\hline & & \multicolumn{9}{c}{ Sin Impacto } & \multicolumn{2}{c}{ Con Impacto } & & \\
Variables & & $\mathbf{n}$ & $\mathbf{\%}$ & $\mathbf{n}$ & $\mathbf{\%}$ & OR & IC (95\%) \\
\hline Edad & 20 a 40 Años & 55 & 67,1 & 60 & 58,3 & & \\
& 41 a 64 Años & 27 & 32,9 & 43 & 41,7 & 1,46 & $(0.79-2.67)$ \\
& & & & & & & \\
\multirow{2}{*}{ Sexo } & Masculino & 51 & 62,2 & 65 & 63,1 & & \\
& Femenino & 31 & 37,8 & 38 & 36,9 & 0,96 & $(0.52-1.75)$ \\
& TOTAL & 82 & 44,32 & 103 & 55,67 & &
\end{tabular}

\section{DISCUSIÓN}

En este estudio se asociaron los aspectos socio demográficos y la calidad de vida en salud bucal, del personal del municipio de Azogues - Ecuador 2015, para ello se utilizó el instrumento OHIP-14Sp, el que se aplicó a 185 personas. Se evaluaron 116 individuos de sexo masculino y 69 de sexo femenino (tabla 1). Un estudio de CVRSB realizado por Guarnizo (9) en Inglaterra, Gales e Irlanda del Norte, también evaluó las condiciones sociales, sin embargo, la distribución de los pacientes de acuerdo a estas variables fue dife- rente predominando en aquel estudio, los pacientes de 35 a 49 años; respecto al sexo estuvieron distribuidos de manera equitativa, y predominó el grupo con algún grado profesional.

En el presente estudio la mayoría de encuestados respondieron que nunca se sintieron limitados ni físicamente, ni psicológicamente por problemas de salud bucal concordando con los trabajos de Rajagopalachari et al., (10), De la Fuente-Hernández (11) y García-Martín (12). 
Respecto a la dificultad para pronunciar palabras el presente estudio mostró que el $28 \%$ autopercibió problemas de este tipo (tabla 2), lo que supera a los reportados por Rajagopalachari et al., que reportan un $10 \%$ de dificultad (10); esta diferencia puede ser atribuida a que en el presente estudio la muestra se logró entre personas de distintas ocupaciones y profesiones en tanto que el estudio de Rajagopalachari se realizó exclusivamente en policías. De igual forma otro estudio en México reporta un 50\% de dificultad para pronunciar palabras (11), lo que no coincide con los hallazgos del presente estudio, esta diferencia puede deberse a que esta población mexicana fue tomada en un servicio de salud, donde los que acuden tiene algún problema que les aqueja en el momento. Otro aspecto de mayor relevancia fue la preocupación respecto a su boca, dientes o prótesis, mientras que el presente estudio encontró impacto en un $60 \%$ de los trabajadores del municipio de la ciudad de Azogues (tabla 2), Rajagopalachari et al., (10) reportaron solamente un $42 \%$ y De la Fuente Hernández 85\% (11); las diferencias son atribuibles a los distintos tipos de población que se investigó. En cuanto al impacto expresado como incomodidad al comer algún alimento el presente estudio reporta $60 \%$ cifra muy parecida a la que publica De la Fuente Hernández (11) $70 \%$ en población de Adultos mayores que demanda atención odontológica, sin embargo estos dos artículos difieren de Rajagopalachari et al., que presenta $42 \%$ de pacientes con dificultad para comer algún alimento (10), posiblemente este valor inferior se deba a que la población cautiva estudiada de policías tenga características de una salud bucal más controlada.

De otro modo el presente estudio reveló que existió diferencia estadísticamente significativa entre los dominios limitación funcional $(\mathrm{p}=0,040)$, dolor físico ( $\mathrm{p}=0,048)$, obstáculos $(\mathrm{p}=0,002)$ al comparar entre los dos grupos de edad, de igual forma el dominio dolor físico mostró tener diferencia estadísticamente significativa al comparar entre ambos sexos en el grupo de 20 a 40 años $(p=0,024)$ (tabla 3 ); datos similares reporta un estudio en Chile Aubert y cols. (13), donde , la dimensión dolor físico $(\mathrm{p}=0,01)$, resultó tener diferencia estadísticamente significativa con mayor impacto en el sexo femenino; también en el estudio chileno las dimensiones molestias e incapacidad psicológica tuvieron impacto en la CVRSB y mostró diferencia entre ambos sexos $(p=0,03 ; p=0,02$ respectivamente), probablemente eso se deba a que en el estudio de Aubert se consideró un rango de edad más amplio que menciona personas más jóvenes desde los 14 años, lo que marca cierta diferencia en cuanto a autopercepción por situaciones de autoestima y relación social.

En cuanto al impacto que genera la salud bucal sobre la calidad de vida de los encuestados con respecto a la edad y sexo se estimó que el 55,67 \% de ellos registraron impacto, predominando los hombres de 20 a 40 años de edad, sin embargo no se asociaron las variables descritas (OR 1,46 I.C.95\% (0,79-2,67); (OR 0,96 I.C.95\% $(0,52-1,75)$ respectivamente) (tabla 6), ello se asemeja al estudio realizado por Yiengprugsawan donde un 49,2\% de individuos menores de 40 años reportaron sentir impacto en su calidad de vida por situaciones dentales (14), lo cual difiere del estudio en México hecho por De la Fuente-Hernández (11) quien reporta al sexo femenino mayor de 60 años como el grupo con mayor impacto tomando como referencia esta situación se justifica debido a que dicho estudio se realizó en población considerada como adulta mayor de 60 años y más. Por otro lado en la investigación realizada por García Martin (12) los resultados contrastan con el presente artículo ya que se hace evidente que el impacto de las condiciones de salud bucal sobre la calidad de vida de los investigados están asociadas (OR 3,294 I.C.95\%(1,388 $7,819)$ ), esto posiblemente a que en estudio de García Martin (12) se investigaron a gestantes que de alguna manera tienen una sensación de menoscabo en su salud por los diversos cambios fisiológicos propios de su estado.

En general la CVRSB estuvo más afectada en el sexo femenino, datos parecidos obtuvo la investigación de Ingle et al., (15) en la India donde el sexo femenino resultó ser el que tenía alta significancia con respecto a las dimensiones; dolor físico, molestias psicológicas apareciendo acá un tercer punto que es el dominio de incapacidad física que tuvo alta significancia estadística en relación a CVRSB con pacientes de sexo femenino posiblemente esa situación sea atribuida al hecho de que se trabajó con pacientes de asistencia a consulta odontológica.

En este contexto se considera importante realizar este tipo de investigaciones para medir condiciones autopercibidas, así como también en su momento relacionarlas con variables clínicas que aportarían con gran 
validez datos interesantes en el proceso salud enfermedad de la población.

Finalmente se puede concluir que, respecto a la CVR$\mathrm{SB}$, más de la mitad de pacientes reportaron sentir impacto en su calidad de vida. El impacto en la CVRSB no está asociada a las condiciones socioeconómicas en la población estudiada.

\section{Correspondencia:}

Cristina Domínguez Crespo
Correo electrónico:
csdominguezc@ucacue.edu.ec

\section{REFERENCIAS BIBLIOGRÁFICAS}

1. Cabezas-Bernhardt G, González-Andrade F, Paredes-Andrade C. Estado de salud oral en el Ecuador. Rev Odontol Act. 2016;1(3):65-70.

2. Ministerio de Salud Pública del Ecuador. Normas y procedimientos de atención en Salud Bucal: Primer Nivel. Quito: Ministerio de Salud Pública; 2009.

3. Ministerio de Salud Pública del Ecuador. Municipios Saludables en el Ecuador Manual para la certificación de municipios: Orientaciones para autoridades y técnicos municipales. Quito: Ministerio de Salud Pública; 2013.

4. Velázquez-Olmedo LB, Ortíz-Barrios LB, Cervantes-Velazquez A, Cárdenas-Bahena Á, García-Peña C, Sánchez-García S. Calidad de vida relacionada con la salud oral en adultos mayores. Instrumentos de evaluación. Rev Med Inst Mex Seguro Soc. 2014; 52(4):448-56.

5. Pita-Fernandez S. Determinación del tamaño muestral. CAD Aten Primaria. 1996; 3: 138-14. (Fecha de acceso: 21 de abril de 2016)Disponible en: https:// www.fisterra.com/mbe/investiga/9muestras/9muestras2.asp

6. León S, Bravo-Cavicchioli D, Correa-Beltrán G, Giacaman RA. Validation of the Spanish version of the Oral Health Impact Profile (OHIP-14Sp) in elderly Chileans. BMC Oral Health. 2014;14(1):95.
7. Atchison KA, Der-Martirosian C, Gift HC. Components of self-reported oral health and general health in racial and ethnic groups. J Public Health Dent. 1998;58(4):301-8.

8. Batista MJ, Perianes LBR, Hilgert JB, Hugo FN, Sousa M da LR de. The impacts of oral health on quality of life in working adults. Braz Oral Res. 2014;28:0-0. Doi: http://dx.doi.org/10.1590/1807-3107BOR-2014. vol28.0040

9. Guarnizo-Herreño CC, Watt RG, Fuller E, et al. Socioeconomic position and subjective oral health: findings for the adult population in England, Wales and Northern Ireland. BMC Public Health. 2014;14(1):827.

10. Rajagopalachari U, Sonde L, Puranik M. Impact of oral health on quality of life among police personnel in Bengaluru City, India: A cross-sectional survey. J Indian Assoc Public Heal Dent. 2015;13(1):42.

11. De la Fuente-Hernández J, Sumano-Moreno Ó, Sifuentes-Valenzuela C, Zelocuatecatl-Aguilar A. Impacto de la salud bucal en la calidad de vida de adultos mayores demandantes de atención dental. Univ Odontol. 2010;29(63):83-92.

12. García-Martin JM, González-Díaz Á, García-Pola MJ. Impacto de la salud oral en la calidad de vida de la gestante Impact of oral health on the quality of life of pregnant woman. Rev Salud Pública. 2017;19(2):145-52.

13. Aubert J, Sanchéz S, Castro R, Monsalves MJ, Castillo P, Moya P. Calidad de vida relacionada con salud oral en mayores de 14 años en la Comunidad San Juan Bautista, Isla Robinson Crusoe, Chile. Int J Odontostomatol. 2014;8(1):141-5.

14. Yiengprugsawan V, Somkotra T, Seubsman S, Sleigh AC. Longitudinal associations between oral health impacts and quality of life among a national cohort of Thai adults. Health Quality of Life Outcomes. 2013;11:172.

15. Ingle $\mathrm{N}$, Chaly $\mathrm{P}$, Zohara $\mathrm{C}$. Oral health related quality of life in adult population attending the outpatient department of a hospital in Chennai, India. Journal of International Oral Health. 2010; 2(4):45-55.

Recibido: 08-07-2018

Aceptado: 13-11-2018 